\title{
New Insights into the Hemodynamic Blood Oxygenation Level- Dependent Response through Combination of Functional Magnetic Resonance Imaging and Optical Recording in Gerbil Barrel Cortex
}

\author{
Andreas Hess, Detlef Stiller, Thomas Kaulisch, Peter Heil, and Henning Scheich \\ Leibniz Institute for Neurobiology, D-39118 Magdeburg, Germany
}

Fast, low-angle shoot functional magnetic resonance imaging (fMRI), based on the blood oxygenation level-dependent (BOLD) effect, was combined with optical recording of intrinsic signals (ORIS) and 2-deoxyglucose labeling in gerbil barrel cortex. We observed over the activated barrel a positive BOLD signal and increased levels of deoxyhemoglobin and total hemoglobin during each period of prolonged (30 sec) D2 vibrissal stimulation. These data show that the hemodynamic basis of this fMRI signal is not necessarily a washout of deoxyhemoglobin, as generally assumed. Instead, they suggest that a positive BOLD signal can also be caused by a local increase of blood volume, even if deoxyhemoglobin levels are persistently ele-

Functional magnetic resonance imaging (fMRI) has rapidly gained experimental and clinical importance as a tool to explore human brain functions and dysfunctions (Kindermann et al., 1997; Buchbinder and Cosgrove, 1998; Turner et al., 1998; Baumgart et al., 1999; Howseman and Bowtell, 1999; Ugurbil et al., 1999). The current fMRI method, based on the blood oxygenation level-dependent (BOLD) effect (Thulborn et al., 1982; Ogawa et al., 1990; Menon et al., 1993; Frahm et al., 1994), is noninvasive and permits repeated measurements. The method has a temporal and spatial resolution and a signal-to-noise ratio, which are all superior to positron emission tomography. The temporal resolution even allows event-related measurements (Dale and Buckner, 1997; for review, see Josephs and Henson, 1999).

Nevertheless, the exact physiological mechanisms behind the BOLD signal are still unresolved. To obtain a steady BOLD signal at acceptable scanner noise levels, prolonged-typically between 30 and $60 \mathrm{sec}$-stimulation is used in fast, low-angle shoot (FLASH) fMRI (Frahm et al., 1994; Bandettini and Wong, 1997; Scheich et al., 1998). The positive polarity of the strongest signal component is commonly interpreted to reflect a decrease of deoxyhemoglobin because of washout by an increased influx of fresh, oxygenated blood (Menon et al., 1993; Cohen and Bookheimer, 1994; DeYoe et al., 1994; Frahm et al., 1994).

However, because the BOLD signal is sensitive to the ratio of

Received Oct. 25, 1999; revised Feb. 2, 2000; accepted Feb. 3, 2000.

This work was supported by Hochschul Sonderprogramm III and Bundesministerium für Bildung, Wissenschaft, Forschung, und Technologie Grant FKZ 0310960/UP2. We thank Drs. K. Sander and M. Woldorff and three anonymous referees for critical comments on earlier versions of this manuscript. We are grateful to Prof. Buxton for essential discussions and comments on our data.

Correspondence should be addressed to Dr. Andreas Hess, Leibniz Institute for Neurobiology, Brenneckestraße 6, D-39118 Magdeburg, Germany. E-mail: hess@ifn-magdeburg.de.

Copyright (C) 2000 Society for Neuroscience $\quad 0270-6474 / 00 / 203328-11 \$ 15.00 / 0$ vated. We also show that this alternative interpretation is consistent with theoretical models of the BOLD signal. The changes in BOLD signal and blood volume, which are most tightly correlated with the periodic stimulation, peak at the site of neuronal activation. These results contribute to the understanding of the hemodynamic mechanisms underlying the BOLD signal and also suggest analysis methods, which improve the spatial localization of neuronal activation with both fMRI and ORIS.

Key words: optical recording; gerbil; BOLD; fMRI; 2-DG; barrel field; somatosensory cortex; rodent; imaging

deoxyhemoglobin to surrounding intravascular and extravascular water (Ogawa et al., 1990; Boxerman et al., 1995) a positive BOLD signal could be caused not only by deoxyhemoglobin washout from a given voxel but also by an increase of the water fraction around the deoxyhemoglobin molecules in that voxel.

Here we use a new approach to the problem by combining two functional imaging methods, fMRI and optical recording of intrinsic signals (ORIS) (Blasdel and Salama, 1986; Grinvald et al., 1986; Frostig et al., 1990; Bonhoeffer and Grinvald, 1996), using identical schemes of stimulation and correlation analyses. ORIS has a higher spatial and temporal resolution than fMRI and can measure, in a much smaller voxel, changes of total deoxyhemoglobin (HbR; at $605 \mathrm{~nm}$ ) and of total hemoglobin (HbT; oxyhemoglobin plus deoxyhemoglobin; at $577 \mathrm{~nm}$ ) and therefore changes in blood volume.

The examined brain structure in the anesthetized gerbil was an individual barrel in cortex during stimulation of the corresponding whisker on the whiskerpad (Woolsey et al., 1975; ArmstrongJames and Fox, 1987; Yang et al., 1996; Peterson et al., 1998). The location of the stimulated and imaged barrel was verified by 2-fluoro-2-deoxy-D-[ $\left.{ }^{14} \mathrm{C}(\mathrm{u})\right]$-glucose (2-DG) autoradiography and cytochrome oxidase staining.

By comparing the spatial and temporal characteristics of the BOLD response over the barrel with those of the two ORIS signals, we examined whether a positive BOLD signal reflects indeed a washout of deoxyhemoglobin or alternatively a blood volume effect, i.e., a relative change of the intravascular water fraction in a given voxel. The obtained results were scrutinized by means of a net-effect BOLD model (Buxton and Frank, 1997; Buxton et al., 1998) (R. Buxton, personal communication).

\section{MATERIALS AND METHODS}

A BOLD contrast map and two ORIS maps (at 577 and $605 \mathrm{~nm}$ wavelengths) were sequentially recorded in the same individual, thereby 
enabling a direct comparison. Identical schemes of prolonged whisker stimulation, necessary or common for fMRI but not typical for ORIS, were used for fMRI and ORIS. Furthermore, a meaningful comparison of fMRI and ORIS also requires analogous data analysis procedures. We therefore analyzed our data with common statistical procedures that are standard for fMRI but novel for ORIS. Finally, a terminal autoradiographic mapping experiment was conducted in each individual, using the metabolic marker 2-DG and a whisker stimulation paradigm as for fMRI and ORIS, to independently determine the location of the activated barrel in each individual. These locations were further confirmed by cytochrome oxidase staining of the obtained sections.

\section{$f M R I$}

Experimental methods. Five adult Mongolian gerbils were anesthetized with $2 \%$ halothane and fixed in a stereotaxic frame. We chose halothane for anesthesia because the animals had to recover completely after each of the experimental steps outlined above and performed over several days. According to Burdett et al. (1995), halothane has only minor effects on the cerebrovascular regulation. Fujibayashi et al. (1994) reported that under normocapnia, halothane has no effect on the energy metabolism of the brain, at least at the concentration used by us, but other results have been obtained (Lindauer et al., 1993). All vibrissae, with the exception of D2, were clipped just above the skin. The animals were then positioned in the center of the magnet of the scanner and kept anesthetized with halothane. A gentle stream of air, periodically pulsed at $8 \mathrm{~Hz}$, was delivered via a small hollow plastic tube that was appropriately positioned, with defined distance and angle, to only deflect the right D2 vibrissa (by $\sim 11^{\circ}$ in rostrocaudal direction). For functional imaging, this stimulus was presented for periods of $30 \mathrm{sec}$, alternating with $30 \mathrm{sec}$ periods without vibrissal stimulation (block design).

fMRI was performed on a 4.7 T BRUKER Biospec scanner with a free-bore of $20 \mathrm{~cm}$ equipped with an actively RF-decoupled coil system. A whole-body birdcage resonator enabled homogeneous excitation, and a $3 \mathrm{~cm}$ surface coil, located directly above the head to maximize the signal-to-noise-ratio, was used as the receiver. The scanning procedure started with the acquisition of T2-weighted spin echo anatomical reference images (thickness, $1.5 \mathrm{~mm}$; field of view, $2.56 \times 2.56 \mathrm{~cm}$; matrix, $256 \times 256$ voxels; $100 \mu \mathrm{m}$ in-plane resolution) using a rapid acquisition relaxation enhanced sequence (RARE) (Hennig et al., 1986). Frontal images were first acquired to identify the rostrocaudal position of the barrel cortex followed by oblique parasagittal images (at an angle of $48^{\circ}$ relative to vertical) to enable a top view onto the left barrel cortex. Corresponding functional images (same thickness, same field of view; matrix, $64 \times 64$ voxels, and hence $400 \mu \mathrm{m}$ in-plane resolution) were collected using a FLASH gradient echo sequence with repetition timeecho time flip angle of $96.7 \mathrm{msec} / 20 \mathrm{msec} / 15^{\circ}$. This parameterization gave a small flip angle, resulting in hardly any flow artifacts (Frahm et al., 1994). Each functional imaging series consisted of 100 successive scans, each of which generated three images at consecutive depths from the cortical surface. Acquisition time for one scan was $6 \mathrm{sec}$, so that the total duration of each series was $600 \mathrm{sec}$. The initial five scans covered a $30 \mathrm{sec}$ period without vibrissal stimulation (no-stimulation period), and the following five scans covered the first $30 \mathrm{sec}$ of vibrissal stimulation (stimulation period). The next five scans covered the second $30 \mathrm{sec}$ no-stimulation period, and so on.

Data analysis. Statistical analysis was performed using a custom-made software package (KHORFu; Gaschler et al., 1997). For each voxel, the time course of the changing amplitude of the BOLD signal over the 600 $\mathrm{sec}$ duration of the experimental series was correlated directly, i.e., without time-lag, with the block-design of stimulation. The resulting $r$ values of the Pearson's correlation coefficients were transformed to $t$ values by a Fisher transformation and subsequently subjected to a Student's $t$ test (Bandettini et al., 1993) with the null hypothesis of no significant correlation with the stimulation paradigm. From these data, two-dimensional maps of correlation coefficients and of levels of significance were generated.

These data were also used to quantify the size of the area activated by the vibrissal stimulation. The size was defined as the product of the in-plane voxel area (viz., $400 \times 400 \mu \mathrm{m}^{2}$ ), and the number of voxels over the barrel cortex for which the change in the signal was correlated with the vibrissal stimulation above selected levels of significance. Commonly, levels of $p<10^{-3}-10^{-4}$ are used in fMRI (Bandettini et al., 1993; Friston et al., 1994; Menon et al., 1997; Scheich et al., 1998), but here we examined the effect of the level of significance more systematically.

The mean time course across experiments and animals was calculated after standardization of each individual time course in terms of means and SDs (see Fig. 4).

\section{ORIS}

Experimental methods. On the day after the fMRI experiments the same animals were reanesthetized with halothane and prepared for optical recording of intrinsic signals from the left barrel cortex. For this purpose, a small aluminum bar, which served as an anchor for head fixation, was mounted to the frontal skull with dental acrylic. The bone over the barrel cortex was thinned. Recording of signals through the thinned bone was performed, under continued anesthesia, with an Imager 2001 System (Optical Imaging). The cortex was homogeneously illuminated by passing light from a DC-regulated tungsten lamp through two fiber optic light guides. Initially, and for reference purposes, an image of the cerebral vascularization pattern was recorded from each animal with light bandpass-filtered at $540 \pm 20 \mathrm{~nm}$. Next, intrinsic signals were recorded using a procedure of vibrissal stimulation identical to that described above for fMRI. For each animal, light that was bandpass-filtered at $577 \pm 6 \mathrm{~nm}$ (ORIS-577) or at $605 \pm 10 \mathrm{~nm}$ (ORIS-605) was used in different recording sessions. Five repetitive experiments per wavelength were performed. The optical system (Ratzlaff and Grinvald, 1991) was defocused approximately down to cortical layer $4( \pm 20 \mu \mathrm{m})$ so that all signals were recorded from the same depth to exclude potential effects caused by the fact that a longer wavelength can derive intrinsic signals from deeper within the cortex. Signals were recorded with a temporal resolution of 1 frame/sec.

Data analysis. The elements of ORIS images are traditionally referred to as pixels, but here we will refer to them as voxels because ORIS, like fMRI, measures signals originating from a cortical volume underneath a small area on the cortical surface.

The ORIS data were analyzed in two ways. First, conventional single condition (i.e., vibrissal stimulation relative to no stimulation) integration maps were calculated for both wavelengths (integration analysis). For this purpose, a first-frame analysis was performed: the data obtained from the first frame of each stimulation or no-stimulation period were subtracted from those of all 29 subsequent frames of that period. These differences were then divided by the data of the same first frame of that period. This procedure reduces the effects of slow nonspecific changes of activity in the cortex during the experiment on the data (Bonhoeffer and Grinvald, 1996). Thereafter, all first-frame corrected data for the five repetitions $\times 10$ presentations of the no-stimulation and the stimulation periods were averaged separately. Integration maps were calculated by subtracting the average no-stimulation image from the stimulation image. These maps were convolved by a $3 \times 3$ Gaussian filter to further improve the signal-to-noise ratio. For display, values between the 10 and $90 \%$ quantiles of the distribution of voxel values were color-coded (integration maps). Integration maps do not depict dynamic temporal changes or statistical coefficients but they accentuate the net direction and spatial extent of the signal changes independent of the temporal changes within each condition.

The second, novel way, in which the ORIS data were analyzed, was by correlating the changing time course of the signal of each voxel with the block-design of stimulation, in the same way as is described above for fMRI (correlation analysis). For ORIS, the data from six consecutive frames were averaged to deliberately reduce the higher temporal resolution of ORIS (viz., 1 frame/ $1 \mathrm{sec}$ ) to that of the fMRI (viz., 1 scan $/ 6$ sec). Maps of correlation coefficients and of levels of significance (correlation maps) were generated, and the size of the activated areas quantified, as described above for fMRI. Average time courses of ORIS signals were computed from voxels for which $p<10^{-8}(605 \mathrm{~nm})$ and $p<$ $10^{-18}(577 \mathrm{~nm})$ and standardized to their mean and SDs (see Fig. 4). For these levels of significance the number of voxels for ORIS-605 and ORIS-577 were most similar (see also Fig. 3).

\section{2-DG autoradiography}

The routine protocol for 2-DG autoradiographic mapping is described in detail elsewhere (Scheich et al., 1993; Hess et al., 1998; Richter et al., 1999). In brief, after $24 \mathrm{hr}$ of recovery after the ORIS experiments, the same animals were reanesthetized with halothane and injected intraperitoneally with $18 \mu \mathrm{Ci}$ of the radioactive metabolic marker 2-DG (American Radiolabeled Chemicals, St. Louis, MO) in $0.2 \mathrm{ml}$ of sterile physiological saline. After $50 \mathrm{~min}$ of vibrissal stimulation, identical to that described above for fMRI, in a dark and sound-attenuating chamber the 

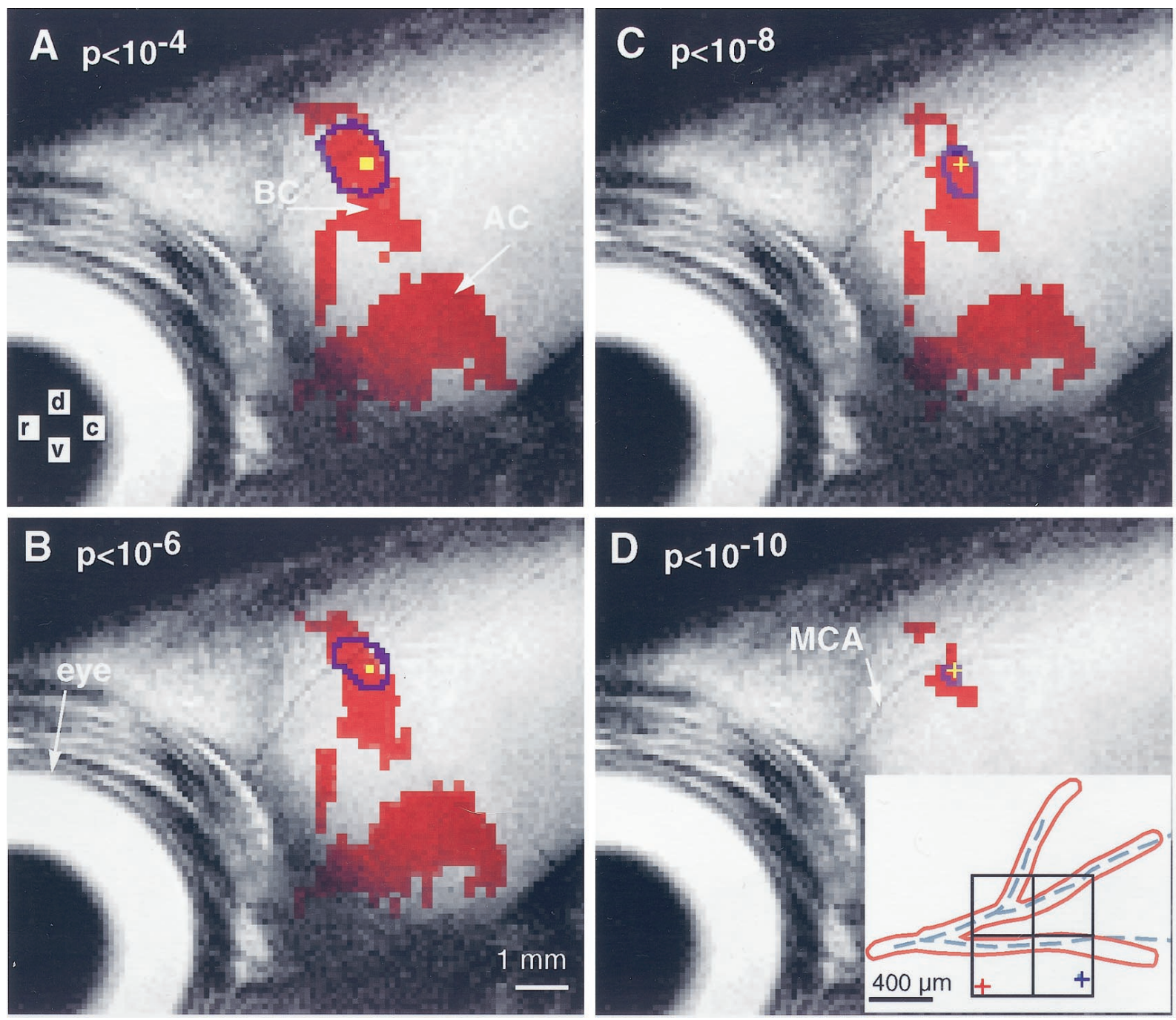

Figure 1. Lateral view of the left cerebral hemisphere from just behind the eye of one gerbil, comprising the barrel cortex ( $B C$ ) and the auditory cortex $(A C)$. The course of the middle cerebral artery $(M C A)$ and its major branches can also be seen. Superimposed onto this anatomical MRI image are shown the location of fMRI voxels (filled in red), of ORIS-577 (outlined in blue), and of ORIS-605 voxels ( $C$, $D$, filled in yellow or yellow crosses) for which the time course of the changing signals was significantly correlated with the block design of vibrissal stimulation. Only voxels with a positive correlation above the level of significance, identified in the top left-hand corner of each chart, are shown. Note that for both methods, the labeled areas of the barrel field shrink with increasing threshold level of significance $(A-D)$. The inset in $D$ shows the alignment of the BOLD and ORIS-577 maps by means of the branching pattern of the MCA, which is identifiable with both methods, and the close match of the centers of gravity of the smallest coherent labeled areas (red cross, fMRI; blue cross, ORIS-577). Note that the mismatch in the location of these centers is less than the in-plane size of two fMRI voxels (squares in inset with $400 \times 400 \mu \mathrm{m}$ each).

animals were killed (T61; Hoechst), and the brains were removed and rapidly frozen on a cryostat. To verify activation of the same barrel with 2-DG, fMRI, and ORIS, a 00 size insect pin was inserted into the cortex $\sim 500 \mu \mathrm{m}$ dorsal to the position of the ORIS-605 labeled area. In the frozen brain lying on its base, this can be done with a micromanipulator under some magnification because even small filled blood vessels are distinguishable on the brain surface and can be matched with vascular images obtained during the ORIS experiments. The pin was driven through the cortex at an angle aimed to intersect the radial orientation of the assumed 2-DG-labeled barrel at some depth. Subsequently, $40 \mu \mathrm{m}$ horizontal sections were cut with a microtome. The sections were mounted on slides and air-dried on a hotplate $\left(50^{\circ} \mathrm{C}\right)$. Thereafter, the sections were exposed to a Kodak (Eastman Kodak, Rochester, NY) NBT-3 x-ray film for $10 \mathrm{~d}$. The $\mathrm{x}$-ray film was developed following standard procedures. The tract of the insect pin could be easily followed through the sections and had a course close to or intersecting the labeled barrel. Sections were then stained for cytochrome oxidase, which delineates barrels (Wong-Riley and Welt, 1980).

\section{RESULTS}

Comparison of gross spatial features of BOLD, ORIS, and 2-DG signals

In this section, the superimposed activation patterns of the stimulated barrel as reflected by the BOLD and ORIS methods will be compared using vascular landmarks as a reference for alignment of the two maps. The activation of the barrel was verified post hoc with the 2-DG mapping method, using the same landmark and with cytochrome oxidase histology.

Figure 1 shows an anatomical MRI image from one animal of a lateral view of that part of the left cerebral hemisphere that comprises the barrel cortex and the auditory cortex caudal to the eye. The course of the middle cerebral artery (MCA) and its major branches can also be clearly seen. Superimposed onto the anatomical image are shown, in red, the location of fMRI voxels 
for which the time course of the changing BOLD signal was significantly correlated with the block design of stimulation of the single D2 vibrissa. In each inset, the distribution of voxels with correlation coefficients above a particular level of significance are shown. The lowest level of significance illustrated, viz., $p<10^{-4}$ (Fig. $1 A$ ), is in the range of conventional fMRI (Bandettini et al., 1993; Friston et al., 1994; Menon et al., 1997; Scheich et al., 1998). Here, a large area around and including the barrel cortex is labeled, viz., $13.12 \mathrm{~mm}^{2}$. This area could accommodate some 460 barrels. The major axis of this area runs approximately from dorsal to ventral, roughly perpendicularly to the rostrocaudally oriented rows of barrels (Woolsey and Welker et al., 1975). With increasing criterion for significance, the labeled area of the barrel cortex shrinks, viz., from $13.12 \mathrm{~mm}^{2}$ (82 voxels) at $p<10^{-4}$ (Fig. $1 A)$ to $3.04 \mathrm{~mm}^{2}$ (19 voxels) at $p<10^{-10}$ (Fig. $1 D$ ).

A smaller rostral patch, ventral to the main barrel cortex, is also labeled at lower levels of significance. This patch may reflect activation of secondary somatosensory cortex SM II (Paxinos, 1995) and/or SM III (Krubitzer et al., 1986). At all levels of significance, large areas of auditory cortex (AC) are also labeled. The location and spatial organizational features of gerbil auditory cortex have been previously studied with electrophysiology, 2-DG, and ORIS (Scheich et al., 1993; Thomas et al., 1993; Hess and Scheich, 1996). The coactivation of auditory cortex was likely caused by the fact that the air puffs used to deflect the D2 vibrissa also produced a noise-like sound.

Figure 1 also shows, outlined in blue, the locations of ORIS-577 voxels over the barrel cortex for which the time course of the changing signal was significantly correlated with the block design of stimulation. Voxels are shown at the same level of significance as for the fMRI data. The BOLD and ORIS-577 maps were aligned by means of the branching pattern of the MCA, which is identifiable with both methods (Fig. $1 D$, inset). At each of the levels of significance, the labeled areas above the barrel cortex differ for BOLD and ORIS-577 with respect to size and shape, being smaller and more concentric for ORIS-577. Nevertheless, the location of the peaks of the stimulation-correlated activation seen with the two methods is remarkably similar. This is illustrated in the inset of Figure $1 D$, where the two crosses identify the location of the centers of gravity (mean of $x$ and $y$ coordinates) of the smallest coherent labeled areas over the barrel cortex ( 5 voxels at $p=10^{-12}$ with fMRI and 50 voxels at $p=$ $10^{-15}$ with ORIS-577 in this animal). The difference in the positions of the two centers of gravity is less than twice the 400 $\mu \mathrm{m}$ in-plane axis of one fMRI voxel (Fig. $1 D$, inset, four squares).

Corresponding data for ORIS-605 voxels are shown in yellow in Figure $1, A$ and $B$. The areas labeled are much smaller than with both fMRI and ORIS-577, and with the higher levels of significance in $C$ and $D$ are much too small to be visible at this image magnification. Therefore, in these latter insets only the positions and not the extent of the ORIS-605 peaks of activation is marked by yellow crosses.

The ORIS-605-labeled area corresponded to the activated barrel, as demonstrated in Figure 2. In this horizontal 2-DG autoradiograph, the activated barrel appears as a darkly labeled radial column extending through the cortical layers and was identified by the lesion, as explained in Materials and Methods. Reduced 2-DG labeling in adjacent nonstimulated barrel cortex was also evident. Such reduced labeling, which has been observed previously in other rodents, may be considered as pericolumnar inhibition (Kossut et al., 1988; Welker et al., 1992) or, alternatively, as an absence of driving activity from the periphery (McCasland

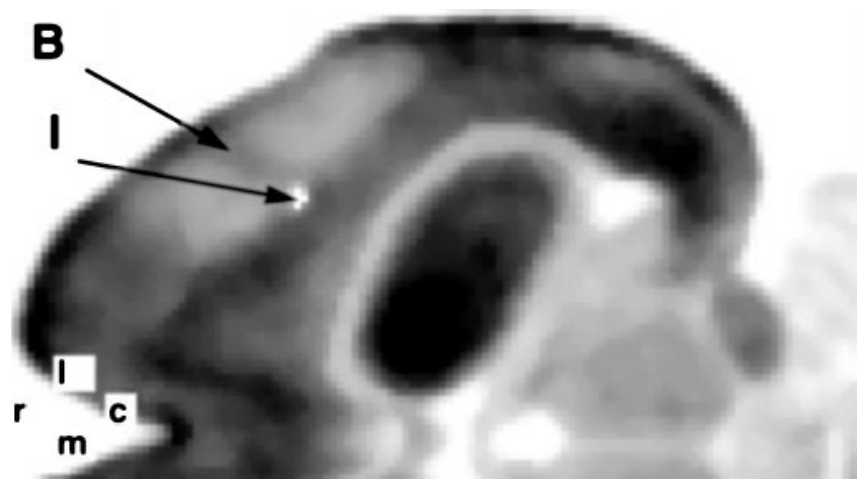

Figure 2. Representative 2-DG autoradiograph of a horizontal section through the barrel cortex. Note the darkly labeled column that reflects the activation of a cortical barrel $(B)$ caused by stimulation of the contralateral D2 vibrissa. The white dot (I) marks the track of the insect pin stuck into the cortex just dorsal to the ORIS-605-labeled area and at an angle to penetrate the cortex beneath that area on the surface. Also note that this track intersects the 2-DG-labeled cortical barrel. From this alignment, it follows that the ORIS-605-labeled area must be in register with the activated cortical barrel.

and Hibbard, 1997). Table 1 provides an anatomical (cytochrome oxidase) and functional (2-DG, ORIS, fMRI) comparison of barrel sizes in rat and gerbil based on our data and data from the literature.

The quantifiable spatial relationships of the three signals are shown for the five animals in Figure 3. For BOLD, ORIS-577, and ORIS-605, the sizes of the coherently activated areas over the barrel cortex are plotted as functions of the level of significance of the correlation with the block design of vibrissal stimulation. The data represent the mean values and SEs across the five animals. For all three signals, the size of the area decreases with increasing level of significance, as would be expected and as was already obvious in Figure $1 A-D$. With high levels of significance $(p<$ $10^{-15}$ ), the BOLD function takes a horizontal course, because here the size of the activated area equals that of the in-plane voxel, whereas the ORIS-577 function continues to decline. Note that, apart from this "ceiling" effect on the BOLD function, the BOLD and ORIS-577 functions are nearly parallel over many orders of magnitude of level of significance. This is in contrast to the course of the function for ORIS-605, which declines much more steeply and reaches the in-plane size of an ORIS voxel (which is more than three orders of magnitude smaller than that of an fMRI voxel) near a level of significance of $p=10^{-15}$.

\section{Comparison of temporal features of BOLD and ORIS signals}

The BOLD and the ORIS-577 signals also share similar temporal characteristics. Figure 4 shows the mean time course, averaged across the five animals, of the BOLD, of the ORIS-577, and of the ORIS-605 signals, computed from voxels for which $p<10^{-5}$. For this level of significance, the areas comprise $\sim 15 \mathrm{~mm}^{2}$ (BOLD), $3 \mathrm{~mm}^{2}$ (ORIS-577), and $0.15 \mathrm{~mm}^{2}$ (ORIS-605) (compare Fig. 3). Whereas all three signals change periodically with a $60 \mathrm{sec}$ period, emphasizing the correlation of the signals with the block design of vibrissal stimulation, the BOLD signal is more similar to the ORIS-577 than to the ORIS-605 signal. This is documented by a higher absolute value of the cross-correlation coefficient between the BOLD and the ORIS-577 signal, viz., -0.80 , than between the BOLD and the ORIS-605 signal, viz., -0.64 . It is important to note that the polarity of the ORIS-605 signal during the first and 
Table 1. Size of a cortical barrel as reflected by different imaging techniques in rat and gerbil

\begin{tabular}{|c|c|c|c|c|}
\hline \multicolumn{2}{|l|}{ Rat (D1) } & & \multicolumn{2}{|l|}{ Gerbil (D2) } \\
\hline $\begin{array}{l}\text { Ratio with cyto- } \\
\text { chrome oxidase }\end{array}$ & $\begin{array}{l}\text { Absolute size } \\
{\left[\mathrm{mm}^{2}\right]}\end{array}$ & & $\begin{array}{l}\text { Absolute size } \\
{\left[\mathrm{mm}^{2}\right]}\end{array}$ & $\begin{array}{l}\text { Ratio with cyto- } \\
\text { chrome oxidase }\end{array}$ \\
\hline & 0.35 & Cytochrome oxidase & 0.092 & \\
\hline \multirow[t]{2}{*}{5.57} & 1.95 & ORIS-605 & 0.518 & 5.63 \\
\hline & & ORIS-577 & 4.211 & 45.77 \\
\hline \multirow[t]{3}{*}{0.57} & 0.2 & $2 \mathrm{DG}$ & 0.056 & 0.61 \\
\hline & & fMRI $10^{-4}$ & 13.12 & 142.60 \\
\hline & & fMRI $10^{-10}$ & 3.04 & 33.00 \\
\hline
\end{tabular}

Data for rat D1 are from Chen-Bee et al. (1996) and for gerbil D2 from the present study. To enable a comparison, the sizes for ORIS-605 and ORIS-577 were obtained with conventional integrative analysis with scaling and clipping procedures given by Chen-Bee et al. (1996). For fMRI, a conventional level $\left(p<10^{-4}\right.$, cf. Fig $\left.1 A\right)$ and a high level of significance $\left(p<10^{-10}\right.$, cf. Fig $1 D$ ) are given. Note the similar ratios of ORIS-605 and of 2DG to cytochrome-oxidase in both species. Moreover, already at a level of significance of $p<10^{-10}$ the area of the fMRI signal is somewhat smaller than that of the conventionally analyzed ORIS-577 signal.

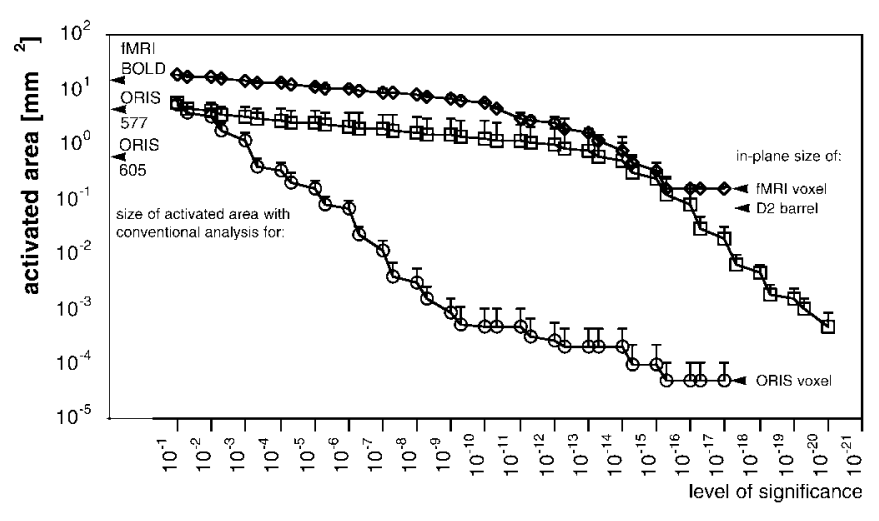

Figure 3. Size of the coherent areas over the barrel field activated by vibrissal stimulation plotted as a function of the level of significance of the Pearson's correlation coefficient for the fMRI BOLD signal and for the ORIS-577 and ORIS-605 signals. The data points represent the means $( \pm$ $\mathrm{SE})$ from the five animals. Note the rather parallel functions for the BOLD (diamonds) and the ORIS-577 signal (squares). For comparison, the sizes of the areas obtained with conventional analysis of fMRI and ORIS data (Masino et al., 1993; Chen-Bee et al., 1996), the in-plane size of fMRI and ORIS voxels, and the size of the D2 barrel in the gerbil are also identified. Note that the latter is smaller than the size of a single fMRI voxel.

all subsequent $30 \mathrm{sec}$ periods of vibrissal stimulation is negative and does not change its polarity during stimulation periods (Fig. $4 D$ ), indicating an elevated deoxyhemoglobin level throughout these periods.

\section{Comparison of detailed spatial features of ORIS signals}

The following comparison of ORIS signals at the wavelengths of 577 and $605 \mathrm{~nm}$ demonstrate in more spatial detail than Figure 1 that voxels of maximum blood volume increase (HbT) correspond to voxels of maximum deoxyhemoglobin increase (HbR), i.e., to the site of neuronal activation. It will also become evident that this correspondence is most clearly seen with correlation analysis of ORIS data, i.e., with the method adopted from fMRI studies, and less so with the conventional analysis of ORIS data, which uses integration of signals and subtraction of nonstimulation from stimulation periods. Because of the complexity of the matter the description of results closely follows those obtained from the case shown in Figure 5 (case different from that in Fig. 1), but the general statements apply to all cases.
Figure 5 shows a comparison of ORIS-605 maps (HbR; Fig. $5 A$ ) with ORIS-577 maps (HbT; Fig. $5 B$ ) and with the pattern of arterial (red) and venous (blue) blood vessels in the area of interest (Fig. 5C), all recorded in the same animal. For the two signals, the conventional integration maps are shown as twodimensional displays of color-coded signal intensities at the bottom of each cube in $A$ and $B$. The pseudo-three-dimensional landscape displays in the cubes show the corresponding maps of correlation coefficients of signals, and the contour maps at the top of the cubes show corresponding isosignificance level contours derived from the correlation coefficients.

The ORIS-605 integration map (Fig. 5A, bottom) reveals several foci of high $\mathrm{HbR}$ (red) in response to the vibrissal stimulation. The focus close to the center of the area of interest (labeled with a white dot) was identified to be over the activated barrel (for method, see Fig. 2), whereas several other more peripherally located foci (labeled with number signs) are placed over draining veins (compare with Fig. 5C).

The distribution of high signal intensities is quite different in the ORIS-577 integration map (Fig. 5B, bottom). This map reveals an increase of $\mathrm{HbT}$ (red) over a relatively large region within the area of interest, which includes the activated barrel (dotted vertical line), but high signal intensities spread extensively in a rostroventral direction toward the origin of the branches of the middle cerebral artery, i.e., upstream (compare Fig. 5C). Downstream over draining veins there is no increase, and in some regions there is even a decrease of $\mathrm{HbT}$ (blue).

In summary, the integration maps at $605 \mathrm{~nm}$ show several foci of high deoxyhemoglobin accumulation, one of which corresponds to the activated barrel. At $577 \mathrm{~nm}$, the increase of total hemoglobin is widespread around the activated barrel and does not allow to identify that barrel. Apart from some other spatial details, which seem important in the context of the long-term stimulation (see Discussion), these reflections of activation correspond to the situation that has been reported in numerous other studies in other cortices and with short-term stimulation.

The novel correlation analysis of the ORIS-605 and ORIS-577 data provide further information on spatial and intensity aspects of ORIS signals and consequently on the variables that determine the BOLD signal. For ORIS-605 (Fig. 5A, landscape), the map of correlation coefficients shows a single sharp peak over the activated barrel, at the same position as the center focus in the integration map described above (dotted vertical line). Typically, correlation coefficients at the positions of the foci over the drain- 


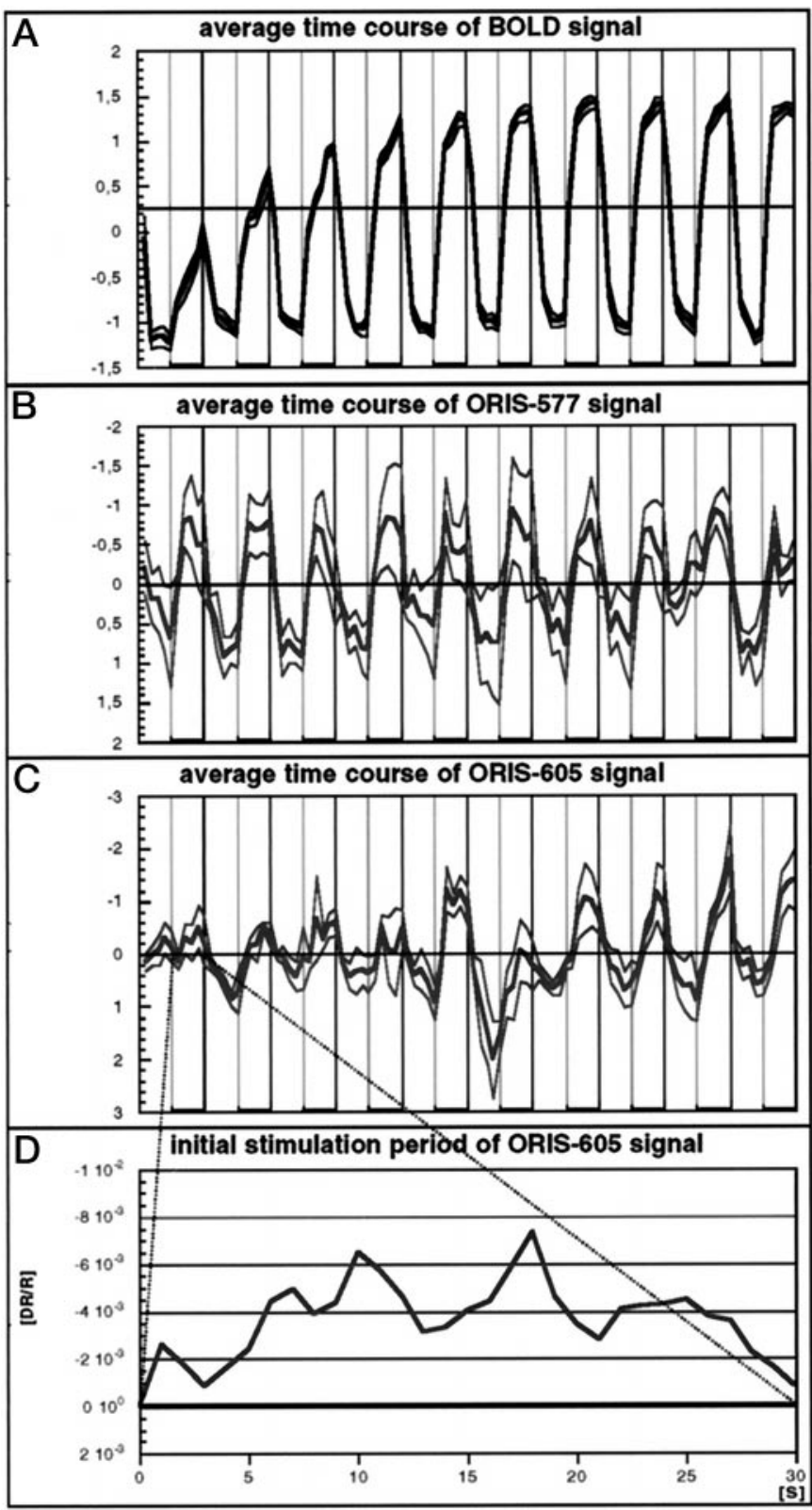

Figure 4. $A-C$, Time course of the standardized BOLD $(A)$, the ORIS$577(B)$, and the ORIS-605 (C) signals, computed for voxels for which $p<$ $10^{-5}$ (BOLD), $p<10^{-8}$ (ORIS-605), and $p<10^{-18}$ (ORIS-577) and averaged across the five animals (mean $\pm \mathrm{SE}$ ). Vertical lines also mark the transition from $30 \mathrm{sec}$ periods of stimulation (thicker horizontal lines) to periods without stimulation. Note that all three signals change periodically with a $60 \mathrm{sec}$ period, i.e., they are correlated with the block design of vibrissal stimulation. Yet, the BOLD signal is more similar to the ORIS577 than to the ORIS-605 signal, as reflected in the absolute value of the cross-correlation coefficients ( -0.80 between BOLD and ORIS- 577 compared to -0.64 between BOLD and ORIS-605). D, Blow up of the ORIS-605 signal during the first $30 \mathrm{sec}$ period of vibrissal stimulation. Note that the signal has the same (negative) polarity throughout that period, reflecting a sustained increased level of $\mathrm{HbR}$.

ing veins in such correlation maps (labeled with number signs) were 10-20\% lower than that over the activated barrel. The map of isosignificance level contours (Fig. $5 \mathrm{~A}$, top) demonstrates that voxels with correlation coefficients of highest significance are also located over the activated barrel (dotted vertical line).

Interestingly, the map of correlation coefficients for the ORIS-
577 data in Figure $5 B$ also reveals a maximum. This maximum is well defined despite the large extent of the region, which shows an increase in HbT (compare with the corresponding integration map; Fig. 5B, bottom). The position of this maximum corresponds closely to that in the corresponding ORIS-605 correlation map (Fig. $5 A$ ). In this case, the difference of only $\sim 150 \mu \mathrm{m}$ was less than the average distance between two adjacent barrels $(184 \mu \mathrm{m})$ in the gerbil, and in all cases was considerably smaller than the in-plane size of a fMRI voxel (Fig. 5C, black square). The same holds with respect to the location of the highest level of significance (Fig. 5C, distance between dotted and dashed vertical lines and between red and green dots; compare also with the white isosignificance level contours in the top maps of Fig. $5 A, B)$. These data suggest that there is also a stimulation-correlated increase in $\mathrm{HbT}$, and therefore of blood volume, with a maximum over the activated barrel.

In summary, the correlation analysis shows that the loci where the changes in the ORIS-605 and ORIS-577 signals (HbR and $\mathrm{HbT}$, respectively) are maximally correlated with the block design of stimulation are well defined and closely correspond to each other. The increase of HbR over the activated barrel (Fig. $5 A$, bottom), which obviously persists throughout each $30 \mathrm{sec}$ stimulation period (Fig. $4 D$ ), suggests that stimulus-induced cortical activation does not necessarily cause a secondary decrease of $\mathrm{HbR}$ because of washout. Therefore, the positive BOLD signal that we have measured in all cases over the activated barrel (Figs. $1,3,4)$ cannot be attributable to HbR washout. The concomitantly elevated $\mathrm{HbT}$ at this site suggests that a blood volume increase may play a role in determining the polarity of the BOLD signal. The fact that $\mathrm{HbR}$ is also somewhat elevated over draining veins as shown by the integration maps suggests that $\mathrm{HbR}$ is washed away from the site of neuronal activation, although at a rate that is not high enough to reduce the HbR concentration over the activated barrel to, or even below, resting levels.

\section{DISCUSSION}

\section{Selective mapping of barrels}

The activation of a cortical barrel by stimulating the corresponding vibrissa can be reliably demonstrated with a variety of techniques. Activity with an approximate spatial dimension of a barrel has previously been demonstrated electrophysiologically (Welker, 1976; Armstrong-James and Fox, 1987; Goldreich et al., 1998; Peterson et al., 1998), with 2-DG (Kossut et al., 1988), and with ORIS at $605 \mathrm{~nm}$ but not at $577 \mathrm{~nm}$ (Masino et al., 1993; Peterson et al., 1998). With conventional FLASH fMRI methods at 7T and single-vibrissa stimulation, Yang et al. (1996, 1997) reported foci of activation with diameters of $360 \pm 215 \mu \mathrm{m}$ corresponding to the area of increased neuronal activity recorded by Armstrong-James and Fox (1987). Our data do not confirm such a correspondence for a positive BOLD signal with conventional statistical criteria. Here, the activated area covered about 460 barrels but decreased to near the size of a single barrel with criteria several decades higher.

\section{Defining a site of neuronal activation with fMRI and ORIS}

With standard statistical criteria for fMRI and conventional integration analysis of ORIS data, it appears that only the ORIS605 signal (HbR) so far has provided the spatial resolution high enough to delineate the locus of neuronal activity (Frostig et al., 1990; Malonek et al., 1997). However, our comparison using correlation analysis of both signals and higher statistical criteria 


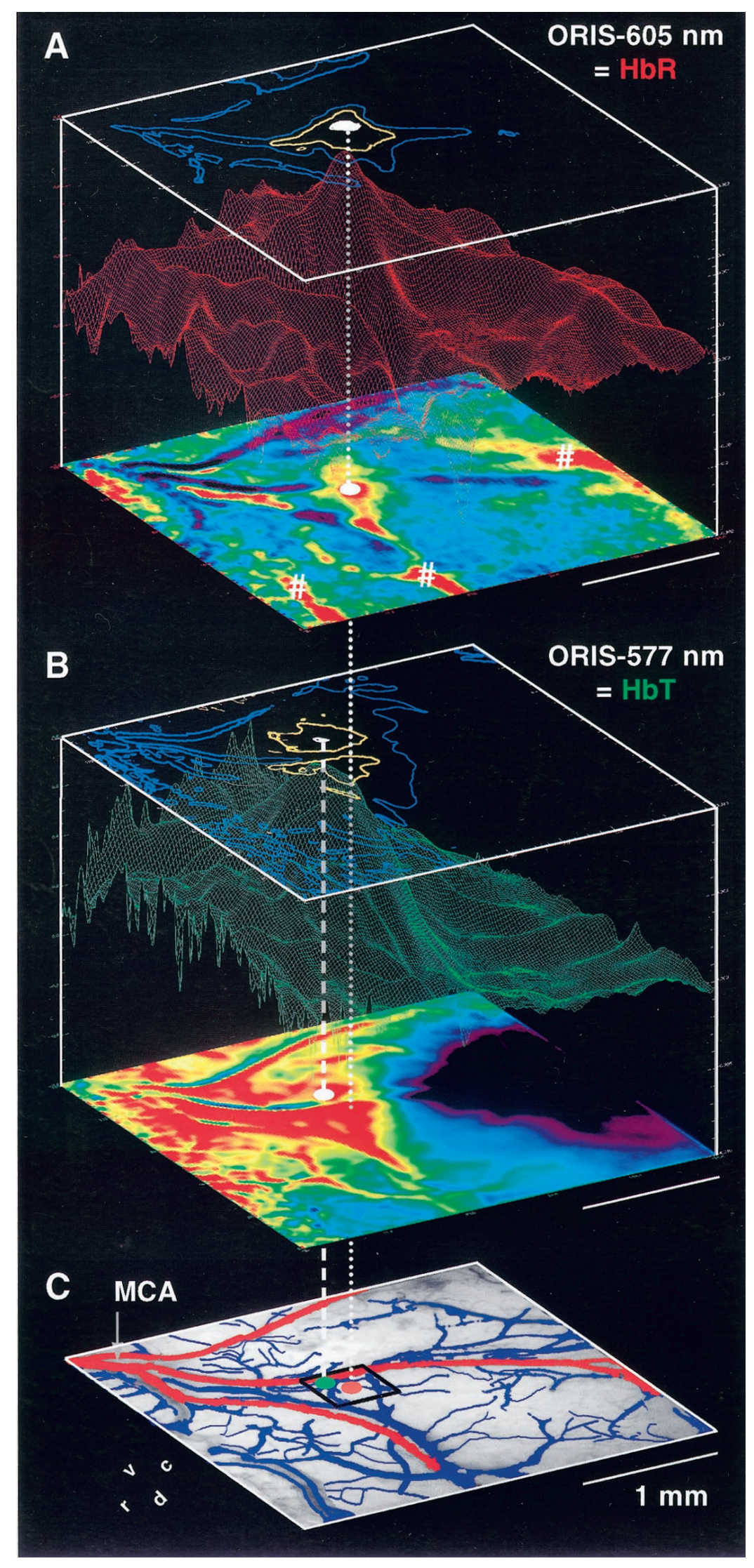

Figure 5. Comparison of maps of ORIS-605 (i.e., $A, \mathrm{HbR}$ ) with ORIS-577 (i.e., $B, \mathrm{HbT}$ ) and with the pattern of blood vessels in the area of interest $(C)$. In $C$, arterial (including the middle cerebral artery MCA) and venous vessels are shown in red and blue, respectively. All maps were recorded in the same animal. For each ORIS signal, the conventional single condition integration map is shown as a color-coded twodimensional display at the bottom of each cube in $A$ and $B$, with an increase in the signal from blue to green to red. Maps of Pearson's correlation coefficients and of levels of significance are shown as three-dimensional landscapes in the center and as isosignificance-level contours at the top of each cube, respectively. In $A$, contours of $p=10^{-3}$ (blue), $10^{-6}$ (yellow), and $10^{-9}$ (white) are shown, and in $B$ of $10^{-3}$ (blue), $10^{-10}$ (yellow), and $10^{-19}$ (white). The split in the yellow contour in $B$ is presumably caused by optical effects of the surface artery. The dotted and dashed vertical white lines project the positions of the highest levels of significance of the ORIS-605 and ORIS-577 signals, respectively, onto the vascular image at the bottom. The black square in $C$ marks the in-plane size of a single fMRI voxel, viz., $400 \times 400 \mu \mathrm{m}^{2}$. 
showed that ORIS-577 and fMRI signals may be spatially nearly as well defined as the ORIS-605 signal. This correlation analysis emphasizes the dynamic changes after (periodic) stimulus onsets and offsets rather than the mere differences of integrated activity as emphasized in previous studies (Masino et al., 1993; Bonhoeffer and Grinvald, 1996; Peterson et al., 1998). Correlation analysis may have general advantages even for the spatial resolution of ORIS-605, namely to distinguish the focus of neuronal activation from nearby signal foci that may appear over draining veins with repetitive stimulation. Their lower correlation strength is probably attributable to a longer delay and/or temporal smearing of the venous increase in $\mathrm{HbR}$ compared to that over the neuronal focus.

Our integration analysis of the ORIS-577 signal revealed widespread areas of increased HbT roughly in the area of the activated barrel (Frostig et al., 1990; Malonek et al., 1997). Whereas a similar spatial extent of signal increase was also visible in the ORIS-577 correlation maps, there was a well defined peak centered to the site of neuronal activation. Thus, ORIS studies, which in some laboratories are conducted at wavelengths less optimal for $\mathrm{HbR}$ because of weak signals at $605 \mathrm{~nm}$, may yield a better definition of activation sites with correlation analysis.

Recently, early time components of the BOLD signal acquired with fast but loud echoplanar imaging have yielded a spatial resolution high enough to demonstrate ocular dominance columns in human (Menon and Goodyear, 1999) and iso-orientation domains in cat primary visual cortex (Kim et al., 2000). Our observations suggest that it should also be possible with more comfortable noise-reduced, gradient echo fMRI (Scheich et al., 1998) to determine the focus of neuronal activation more accurately, viz., by raising the criterion for significant correlation. As shown by the high-resolution ORIS method, draining veins do not appear to be a major confounding problem.

\section{Hemodynamics and the BOLD effect}

In the following, some settings and boundary conditions of components that determine the BOLD and ORIS signals are discussed. The conclusions are guided by the model given in the Appendix and apply to our data.

Because the BOLD effect is determined by the total amount of deoxyhemoglobin as well as by the ratio of deoxyhemoglobin to free water within a given voxel (see Appendix), a positive BOLD contrast can theoretically occur under several conditions: (1) if there is a decrease of deoxyhemoglobin caused by an increased influx of fresh oxygenated blood (depletion); (2) if the intravascular water fraction surrounding a given amount of deoxyhemoglobin molecules increases, by an increase in cerebral blood volume (CBV; dilution); and (3) if both the intravascular amount of deoxyhemoglobin and $\mathrm{CBV}$ will increase, but $\mathrm{CBV}$ more strongly. If their ratio remains constant, a negative signal will result because of an increased total amount of dephasing deoxyhemoglobin molecules within the voxel of observation (build-up; see Eq. 3 in the Appendix).

In a voxel with multiple capillaries, all three conditions lead to a positive BOLD signal if the described changes hold in similar ways for all capillaries involved independent of their number. It is possible to measure an elevated HbR level in a voxel, as we did here with ORIS-605, and still measure a positive BOLD signal, as we also did under several circumstances: for instance, if capillaries are widened or more of them are opened or if the ratio of water to red blood cells in capillaries of maintained diameter increases. These hemodynamic changes may all result in an intravascular dilution of the produced deoxyhemoglobin (see Appendix).

Our finding of sustained $\mathrm{HbR}$ increase during repetitive stim- ulation is not the only evidence of such long-term effects. During prolonged stimulation sustained elevated $\mathrm{HbR}$ production and HbR content were also suggested by fMRI experiments comparing the effects of hypercapnia and visual stimulation in visual cortex (Kim and Ugurbil, 1997; Davis et al., 1998; Schwarzbauer and Heinke, 1999). Hypercapnia produced a large increase of cerebral blood flow $(\mathrm{CBF})$ and $\mathrm{CBV}$, presumably without increasing the oxygen consumption. The resulting large, positive BOLD signal was therefore caused by washout and dilution of the "normal" HbR production at rest. Under visual stimulation the BOLD signal was lower than during hypercapnia, but still positive, throughout the stimulation periods ( 2 min). Because CBF was not different in the two conditions, this suggests that the reduction of the positive BOLD signal was attributable to a sustained increase in $\mathrm{HbR}$ production. A maintained $\mathrm{HbR}$ increase during prolonged stimulation has also been concluded from fMRI experiments in motor cortex (Haacke et al., 1997) and was directly shown with infrared spectroscopy in human visual cortex (Kato et al., 1993, 1999).

Findings of sustained HbR increase during prolonged stimulation cannot be directly compared with data obtained with short periods of stimulation. For instance, in ORIS studies using short periods of stimulation (e.g., $2 \mathrm{sec}$ ) a decrease of HbR below baseline ("undershoot") a few seconds after an initial increase of HbR has been reported (Narayan et al., 1994; Cannestra et al., 1996; Malonek et al., 1997). However, in Malonek et al. (1997) (compare Fig. 1E), the undershoot disappeared with two or three such periods of visual stimulation. This suggests that already with somewhat prolonged stimulation the relationships of $\mathrm{HbR}$ production, and changes of local $\mathrm{CBV}$ and $\mathrm{CBF}$ result in an elevated HbR content.

Furthermore, Malonek et al. (1997) found that the early increase of HbR was accompanied by a simultaneous increase of $\mathrm{HbT}$. Because the change of $\mathrm{CBF}$, measured independently by laser-doppler flowmetry, was delayed relative to the $\mathrm{HbR}$ and $\mathrm{HbT}$ increase, the authors concluded that the early change of $\mathrm{HbT}$ probably reflected an increase in $\mathrm{CBV}$, controlled by an early mechanism independent of that controlling the flow component. Similarly, Marota et al. (1999) showed an early and similar onset of the BOLD and the CBV signal. The data of Mandeville et al. (1998), often cited for a slower increase of CBV compared to the BOLD signal after single stimulation, merely show that the CBV signal takes longer to reach its peak value. Such different dynamics do not exclude a causal relationship between the two signals. Similarly, the results of Mandeville et al. (1998) and Marota et al. (1999) in the anesthetized rat brain, showing that the average increase of $\mathrm{CBV}$ in an area is much smaller than the increase of $\mathrm{CBF}$, does not exclude our hypothesis. Focusing on the spatial aspect of signal distributions, we could show a close correspondence of the loci of maximum correlation of ORIS-605 and ORIS-577 signals (Fig. 5) and of the BOLD signal (Fig. 1D), suggesting that the volume effect must be largest at the site of highest HbR content, i.e., at the site of neuronal activation.

Because the local volume increase in the microvessels at that site occurs as early as the HbR increase (Malonek et al., 1997), the above consideration may also shed some light on the "initial dip" (i.e., a negative BOLD polarity) analyzed in some fMRI studies (Menon et al., 1995; Hennig et al., 1997; Hu et al., 1997; Kim et al., 2000). This effect may be caused by special initial conditions in which an increase of $\mathrm{HbR}$ driving the BOLD signal in negative direction is stronger than a concomitant volume increase driving it into positive direction. 
In summary, our results may contribute to the understanding of the hemodynamic mechanisms underlying the BOLD signal over activated brain regions, even though this has been studied only in a specialized cortex and in an anesthetized animal. The results emphasize the influence of the blood volume changes and of the intravascular signal component. Furthermore, we suggest analysis methods to improve the spatial localization of neuronal activation with both fMRI and ORIS.

\section{APPENDIX \\ Theoretical considerations and evaluation of a model of the BOLD signal}

The data presented above bear directly on the mechanisms underlying the BOLD signal, at least during long-term activation of the barrel cortex of the anesthetized gerbil. To follow our reasoning below, two points should be remembered. First, although the BOLD method measures deoxyhemoglobin changes within a voxel of observation, the BOLD effect is determined by the total amount of deoxyhemoglobin as well as by the ratio of deoxyhemoglobin to free water within this voxel. This is so because paramagnetic deoxyhemoglobin molecules act as "sinks" for the relaxation of spins of protons in the water immediately surrounding the deoxyhemoglobin molecules (Pauling, 1977; Thulborn et al., 1982; Ogawa et al., 1990; Kwong and Chesler, 1992; Ogawa et al., 1992). Water inside blood vessels will therefore contribute more to the BOLD contrast than water in surrounding tissue (Boxerman et al., 1995). Second, it should be remembered that the average concentration of deoxyhemoglobin in a given voxel (HbR) is not the same as its concentration within the local volume of blood, the intravascular (blood) concentration, contained in that voxel. Only some fraction of the cortical volume that contributes signals to a fMRI voxel is made up of blood vessels, but those contain all the deoxyhemoglobin. It is conceivable that the intravascular concentration of deoxyhemoglobin is more relevant to the BOLD signal than the average voxel concentration dependent on how short reaching the influence of deoxyhemoglobin molecules on the surrounding water is. In his theory of the basic principles of deoxyhemoglobin as an intrinsic contrast-agent Ogawa (Ogawa and Tank et al., 1992; Ogawa and Menon et al., 1993) assumed that the contribution of the intravascular water to the BOLD signal is small because of the small blood volume fraction in a voxel. This assumption has lately been questioned. Boxerman et al. (1995) showed that the contribution of the intravascular water fraction to the BOLD signal is dominant, at least at lower field strengths, and could cause up to two-thirds of the BOLD signal.

If one considers only the BOLD and the ORIS-577 signals from our data, their near-parallel area functions (Fig. 3) and high absolute value of the cross-correlation coefficient (Fig. 4, compare $B, C$ ) would be consistent with the conventional interpretation, namely that a positive BOLD signal in a voxel of observation is caused by a decrease in the HbR concentration in that voxel because of washout of $\mathrm{HbR}$ from vessels (depletion). This would be caused by an increased CBF during stimulation, also leading to an increase in HbT and hence an increase of the ORIS-577 signal. However, the ORIS-605 signal clearly revealed that the HbR concentrations in all voxels from the activated barrel were persistently elevated during stimulation. For this case, Ogawa's theory, based on the dominance of the extravascular water component, predicts a negative BOLD signal. Therefore, the above findings together are clearly not compatible with the deoxyhemoglobin depletion interpretation of the positive BOLD signal.

To explore these possibilities theoretically, we transformed a basic net effect model of the BOLD signal, which incorporates both the extravascular and the intravascular components (Buxton et al., 1998). It is necessary to consider the BOLD signal as an explicit function of the total deoxyhemoglobin content and of $\mathrm{CBV}$ in a voxel and to pay attention to the contributions of both extravascular and intravascular signal components. A signal evaluation in this required form, valid for low-resolution gradient echo sequences at $1.5 \mathrm{~T}$ and $40 \mathrm{msec}$ echo time, was provided for humans by Buxton et al. (1998) based either on simulation data and relaxation theory or considering experimental values for the apparent transversal relaxation time $\mathrm{T} 2 *$ of blood as a function of oxygenation (Buxton et al., 1998; Buxton, personal communication). According to Buxton, the BOLD signal can be written as:

$$
\frac{\Delta S}{S}=V_{0}\left[\left(k_{1}+k_{2}\right)(1-q)-\left(k_{2}+k_{3}\right)(1-v)\right]
$$

with $V_{0}$ as the blood volume fraction at rest, $v=V / V_{0}$ as the ratio of the current blood volume fraction, $V$ and $V_{0}$ and $q=Q / Q_{0}$ as the ratio of the current total deoxyhemoglobin content $Q$, and the total deoxyhemoglobin content at rest $Q_{0}$. The dimensionless parameters $k_{1,2,3}$ are related to the ratio of extravascular and intravascular signal contribution and their balanced dependence on $q$ and $v$. The numerical values have been calculated by Buxton et al. (1998; Buxton, personal communication) to $k_{1}=2.8, k_{2}=$ 0.57 , and $k_{3}=0.43$.

From the relations:

$$
\frac{\partial}{\partial q}\left(\frac{\Delta S}{S}\right)=-V_{0}\left(k_{1}+k_{2}\right)<0 \quad \frac{\partial}{\partial v}\left(\frac{\Delta S}{S}\right)=V_{0}\left(k_{2}+k_{3}\right)>0
$$

we can derive that for small increases of total deoxyhemoglobin content in a voxel (HbR), CBV must also increase by a constant ratio to compensate the effect of increasing $\mathrm{HbR}$. Then, the condition for a positive BOLD response is described by the relation quantifying the counteracting effects of increasing intravascular and decreasing extravascular contributions:

$$
\left(k_{1}+k_{2}\right) \Delta q<\left(k_{2}+k_{3}\right) \Delta v
$$

It is important to note that this relation implicitly comprises a decreasing concentration of intravascular deoxyhemoglobin in blood because the blood volume fraction has to go up more than the total content of deoxyhemoglobin. This is a hitherto neglected additional aspect of the BOLD effect and suggests that the conventional interpretation that a positive BOLD signal can only be measured for decreasing total deoxyhemoglobin in a voxel (HbR) should be replaced by one that requires a decrease in the intravascular deoxyhemoglobin concentration. This formulation also includes the case of a reduced total deoxyhemoglobin (HbR) content in a given voxel that is also governed by Inequation. Using higher magnetic field strengths would influence the quantitative aspects because the intravascular contribution to BOLD becomes less dominant (Gati et al., 1997). The qualitative aspect, however, is improbable to change unless at extremely high fields.

Equation 1 describes the signal one could expect to measure for a given combination of $q$ and $v$, regardless of which dynamics in oxygen consumption and regulation of $\mathrm{CBF}$ and $\mathrm{CBV}$ can lead to such conditions. It is also possible to choose $\mathrm{CBF}$ and cerebral metabolic rate of oxygen consumption $\left(\mathrm{CMRO}_{2}\right)$ as independent variables to model the BOLD signal, which would eliminate the explicit dependence on CBV (Kim et al., 1999). However, because all these properties are physiologically related, which design to use is a question of suitability and direct availability. 
The new perspective provided by the BOLD effect model with $\mathrm{CBV}$ and $\mathrm{HbR}$ as relatively independent variables may also shed some light on transient effects such as the "initial dip" and the "poststimulus undershoot" described in various reports (Fransson et al., 1997; Hu et al., 1997; Buxton et al., 1998; Fransson et al., 1998; Jones et al., 1998, 1999). With the beginning of an increased neuronal activity causing an increased $\mathrm{CMRO}_{2}$, the local intravascular concentration of deoxyhemoglobin rises. Next, as Malonek et al. (1997) could demonstrate with ORIS, CBV increases. Additionally, $\mathrm{CBF}$ increases. During this initial time window, an initial dip might be measured if the increase in the amount of deoxyhemoglobin dominates over the diluting effect of the starting increase in $\mathrm{CBV}$ and the washout effect of the increasing $\mathrm{CBF}$. Although CBV and CBF increase, this does not necessarily lead to a decrease in the total amount of deoxyhemoglobin in a voxel (HbR). The coupling ratio of $\mathrm{CBF}$ and $\mathrm{CBV}$ may not be constant across different species and might also be affected by anesthesia. For example, it is consistently described for awake humans (Davis et al., 1998; Kim et al., 1999) and other species that CBF rises up to twice as much as $\mathrm{CMRO}_{2}$ and by a constant ratio in humans (Hoge et al., 1999), thus reducing the intravascular deoxyhemoglobin concentration to or below resting level. This reported strong increase of CBF alone would lead to a decreased total amount of deoxyhemoglobin (HbR). However, this finding does not apply to our data. We documented here a persistent increase of HbR over the activated barrel throughout each $30 \mathrm{sec}$ stimulation period, in spite of an increase of CBF in the barrel (Cox et al., 1993). We conclude from this finding that the increase of CBF was not strong enough to wash out the increased production of deoxyhemoglobin.

After stimulation has ceased, $\mathrm{CBF}$ and $\mathrm{CBV}$ decline to baseline levels. The transient effect of the well known poststimulus undershoot, i.e., a negative BOLD signal after stimulus offset (Fransson et al., 1997; Hu et al., 1997; Buxton et al., 1998; Fransson et al., 1998; Jones et al., 1998; Jones, 1999), may occur if the abrupt downregulation of $\mathrm{CBF}$ and a slower readjustment of $\mathrm{CBV}$, with distinct time constants, leads to pooling of deoxyhemoglobin on the venous side. Then a somewhat prolonged generation of deoxyhemoglobin that is not accompanied by a CBV sufficient to compensate for the persistent deoxyhemoglobin concentration could lead to a negative BOLD signal.

To summarize our theoretical considerations, the finding of persistently increased deoxyhemoglobin content in a voxel (HbR) during prolonged stimulation periods is not in contradiction to the common theory of the BOLD signal if intravascular components are considered, i.e., if the intravascular deoxyhemoglobin concentration is diluted by a volume effect.

\section{REFERENCES}

Armstrong-James M, Fox K (1987) Spatiotemporal convergence and divergence in the rat S1 "barrel" cortex. J Comp Neurol 263:265-281.

Bandettini PA, Wong EC (1997) Magnetic resonance imaging of human brain function. Principles, practicalities, and possibilities. Clin Neurosurg 8:345-371.

Bandettini PA, Jesmanowicz A, Wong EC, Hyde JS (1993) Processing strategies for time-course data sets in functional MRI of the human brain. Magn Reson Med 30:161-173.

Baumgart F, Gaschler-Markefski B, Woldorff MG, Heinze HJ, Scheich H (1999) A movement-sensitive area in auditory cortex [letter]. Nature 400:724-726.

Blasdel GG, Salama G (1986) Voltage-sensitive dyes reveal a modular organization in monkey striate cortex. Nature 321:579-585.

Bonhoeffer T, Grinvald A (1996) Optical imaging based on intrinsic signals: the methodology. In: Brain mapping: the methods (Toga AW, Mazziotta JC, eds), pp 55-97. London: Academic.

Boxerman JL, Bandettini PA, Kwong KK, Baker JR, Davis TL, Rosen
BR, Weisskoff RM (1995) The intravascular contribution to fMRI signal change: Monte Carlo modeling and diff usion-weighted studies in vivo. Magn Reson Med 34:4-10.

Buchbinder BR, Cosgrove GR (1998) Cortical activation MR studies in brain disorders. Magn Reson Imaging Clin N Am 6:67-93.

Burdett NG, Menon DK, Carpenter TA, Jones JG, Hall LD (1995) Visualisation of changes in regional cerebral blood flow (rCBF) produced by ketamine using long TE gradient-echo sequences: preliminary results. Magn Reson Imaging 13:549-553.

Buxton RB, Frank LR (1997) A model for the coupling between cerebral blood flow and oxygen metabolism during neural stimulation. J Cereb Blood Flow Metab 17:64-72.

Buxton RB, Wong EC, Frank LR (1998) Dynamics of blood flow and oxygenation changes during brain activation: the balloon model. Magn Reson Med 39:855-864.

Cannestra AF, Blood AJ, Black KL, Toga AW (1996) The evolution of optical signals in human and rodent cortex. NeuroImage 3:202-208.

Chen-Bee CH, Kwon MC, Masino SA, Frostig RD (1996) Areal extent quantification of functional representations using intrinsic signal optical imaging. J Neurosci Methods 68:27-37.

Cohen MS, Bookheimer SY (1994) Localization of brain function using magnetic resonance imaging. Trends Neurosci 17:268-277.

Cox SB, Woolsey TA, Rovainen CM (1993) Localized dynamic changes in cortical blood flow with whisker stimulation corresponds to matched vascular and neuronal architecture of rat barrels. J Cereb Blood Flow Metab 13:899-913.

Dale AM, Buckner RL (1997) Selective averaging of rapidly presented individual trials using fMRI. Human Brain Mapp 5:329-340.

Davis TL, Kwong KK, Weisskoff RM, Rosen BR (1998) Calibrated functional MRI: mapping the dynamics of oxidative metabolism. Proc Natl Acad Sci USA 95:1834-1839.

DeYoe EA, Bandettini P, Neitz J, Miller D, Winans P (1994) Functional magnetic resonance imaging (FMRI) of the human brain. J Neurosci Methods 54:171-187.

Frahm J, Merboldt KD, Hanicke W, Kleinschmidt A, Boecker H (1994) Brain or vein-oxygenation or flow? On signal physiology in functional MRI of human brain activation. NMR Biomed 7:45-53.

Fransson P, Kruger G, Merboldt KD, Frahm J (1997) A comparative FLASH and EPI study of repetitive and sustained visual activation. NMR Biomed 10:204-207.

Fransson P, Kruger G, Merboldt KD, Frahm J (1998) Temporal characteristics of oxygenation-sensitive MRI responses to visual activation in humans. Magn Reson Med 39:912-919.

Friston KJ, Jezzard P, Turner R (1994) Analysis of functional MRI time-series. Human Brain Mapp 1:153-171.

Frostig RD, Lieke EE, Ts'o DY, Grinvald A (1990) Cortical functional architecture and local coupling between neuronal activity and the microcirculation revealed by in vivo high-resolution optical imaging of intrinsic signals. Proc Natl Acad Sci USA 87:6082-6086.

Fujibayashi T, Sugiura Y, Yanagimoto M, Harada J, Goto Y (1994) Brain energy metabolism and blood flow during sevoflurane and halothane anesthesia: effects of hypocapnia and blood pressure fluctuations [corrected and republished article originally printed in Acta Anaesthesiol Scand (1993) 37:806-810]. Acta Anaesthesiol Scand 38:413-418.

Gaschler B, Baumgart F, Tempelman C, Stiller D, Heinze HJ, Scheich H (1997) Statistical methods in functional magnetic resonance imaging with respect to non-stationary time series - auditory cortex activity. Magn Reson Med 38:811-820.

Gati JS, Menon RS, Ugurbil K, Rutt BK (1997) Experimental determination of the BOLD field strength dependence in vessels and tissue. Magn Reson Med 38:296-302.

Goldreich D, Peterson BE, Merzenich MM (1998) Optical imaging and electrophysiology of rat barrel cortex. II. Responses to paired-vibrissa deflections. Cereb Cortex 8:184-192.

Grinvald A, Lieke E, Frostig RD, Gilbert CD, Wiesel TN (1986) Functional architecture of cortex revealed by optical imaging of intrinsic signals. Nature 324:361-364.

Haacke M, Lai S, Reichenbach J, Kuppusamy F, Hoogenraad F, Takeichi $\mathrm{H}$, Lin W (1997) In vivo measurement of blood oxygen saturation using magnetic resonance imaging: a direct validation of the blood oxygen level dependent concept in functional brain imaging. Human Brain Mapp 5:341-346.

Hennig J, Nauerth A, Friedburg H (1986) RARE imaging: a fast imaging method for clinical MR. Magn Reson Med 3:823-833.

Hennig J, Janz C, Speck O, Ernst T (1997) Is there a different type of MR-contrast in the early phase of functional activation? Adv Exp Med Biol 413:35-42. 
Hess A, Scheich H (1996) Optical and FDG mapping of frequencyspecific activity in auditory cortex. NeuroReport 7:2643-2647.

Hess A, Lohmann K, Gundelfinger ED, Scheich H (1998) A new method for reliable and efficient reconstruction of 3-dimensional images from autoradiographs of brain sections. J Neurosci Methods 84:77-86.

Hoge RD, Atkinson J, Gill B, Crelier GR, Marrett S, Pike GB (1999) Investigation of BOLD signal dependence on cerebral blood flow and oxygen consumption: the deoxyhemoglobin dilution model [in process citation]. Magn Reson Med 42:849-863.

Howseman AM, Bowtell RW (1999) Functional magnetic resonance imaging: imaging techniques and contrast mechanisms. Philos Trans R Soc Lond B Biol Sci 354:1179-1194.

Hu X, Le TH, Ugurbil K (1997) Evaluation of the early response in fMRI in individual subjects using short stimulus duration. Magn Reson Med 37:877-884.

Jones RA (1999) Origin of the signal undershoot in BOLD studies of the visual cortex [in process citation]. NMR Biomed 12:299-308.

Jones RA, Schirmer T, Lipinski B, Elbel GK, Auer DP (1998) Signal undershoots following visual stimulation: a comparison of gradient and spin-echo BOLD sequences. Magn Reson Med 40:112-118.

Josephs O, Henson RN (1999) Event-related functional magnetic resonance imaging: modelling, inference and optimization. Philos Trans R Soc Lond B Biol Sci 354:1215-1228.

Kato T, Takashima S, Kamada K, Kishibayashi J, Sunohara N, Ozaki T (1993) Advantage of near-infrared spectroscopy in the human functional MR imaging in brain. Proceedings of the SMRM, 12th annual meeting, New York, August.

Kato T, Yamashita Y, Sugihara K, Furusho J, Tazaki I, Tanaka D, Maki A, Yamamoto T, Koizumi H, Ichikawa N, Iikura Y (1999) Cerebral autonomic functional test using human functional near-infraredgraphy (FNIR). NeuroImage 9:221.

Kim DS, Duong TQ, Kim SG (2000) High-resolution mapping of isoorientation columns by fMRI. Nat Neurosci 3:164-169.

Kim SG, Ugurbil K (1997) Comparison of blood oxygenation and cerebral blood flow effects in fMRI: estimation of relative oxygen consumption change. Magn Reson Med 38:59-65.

Kim SG, Rostrup E, Larsson HB, Ogawa S, Paulson OB (1999) Determination of relative $\mathrm{CMRO} 2$ from $\mathrm{CBF}$ and $\mathrm{BOLD}$ changes: significant increase of oxygen consumption rate during visual stimulation. Magn Reson Med 41:1152-1161.

Kindermann SS, Karimi A, Symonds L, Brown GG, Jeste DV

Kim SG, Ugurbil K (1997) Review of functional magnetic resonance imaging in schizophrenia. Schizophr Res 27:143-156.

Kossut M, Hand PJ, Greenberg J, Hand CL (1988) Single vibrissal cortical column in SI cortex of rat and its alterations in neonatal and adult vibrissa-deafferented animals: a quantitative 2DG study. J Neurophysiol 60:829-852.

Krubitzer LA, Sesma MA, Kaas JH (1986) Microelectrode maps, myeloarchitecture, and cortical connections of three somatotopically organized representations of the body surface in the parietal cortex of squirrels. J Comp Neurol 250:403-430.

Kwong KKB, Belliveau JW, Chesler DA (1992) Dynamic magnetic resonance imaging of human brain activity during primary sensory stimulation. Proc Natl Acad Sci USA 89:5675-5679.

Lindauer U, Villringer A, Dirnagl U (1993) Characterization of CBF response to somatosensory stimulation: model and influence of anesthetics. Am J Physiol 264:H1223-H1228.

Malonek D, Dirnagl U, Lindauer U, Yamada K, Kanno I, Grinvald A (1997) Vascular imprints of neuronal activity: relationships between the dynamics of cortical blood flow, oxygenation, and volume changes following sensory stimulation. Proc Natl Acad Sci USA 94:14826-14831.

Mandeville JB, Marota JJA, Kosofsky BE, Keltner JR, Weissleder R, Rosen BR, Weisskoff RM (1998) Dynamic functional imaging of relative cerebral blood volume during rat forepaw stimulation. Magn Reson Med 39:615-624.

Marota JJ, Ayata C, Moskowitz MA, Weisskoff RM, Rosen BR, Mandeville JB (1999) Investigation of the early response to rat forepaw stimulation. Magn Reson Med 41:247-252.

Masino SA, Kwon MC, Dory Y, Frostig RD (1993) Characterization of functional-organization within rat barrel cortex using intrinsic signal optical imaging through a thinned skull. Proc Natl Acad Sci USA 90:9998-10002.

McCasland JS, Hibbard LS (1997) GABAergic neurons in barrel cortex show strong, whisker-dependent metabolic activation during normal behavior. J Neurosci 17:5509-5527.

Menon RS, Goodyear BG (1999) Submillimeter functional localization in human striate cortex using BOLD contrast at 4 Tesla: implications for the vascular point-spread function. Magn Reson Med 41:230-235.

Menon RS, Ogawa S, Hu X, Strupp JP, Anderson P, Ugurbil K (1995) BOLD based functional MRI at 4 Tesla includes a capillary bed contribution: echo-planar imaging correlates with previous optical imaging using intrinsic signals. Magn Reson Med 33:453-459.

Menon RS, Ogawa S, Tank DW, Ugurbil K (1993) Tesla gradient recalled echo characteristics of photic stimulation-induced signal changes in the human primary visual cortex. Magn Reson Med 30:380-386.

Menon RS, Ogawa S, Strupp JP, Ugurbil K (1997) Ocular dominance in human V1 demonstrated by functional magnetic resonance imaging. J Neurophysiol 77:2780-2787.

Narayan SM, Santori EM, Blood AJ, Burton JS, Toga AW (1994) Imaging optical reflectance in rodent barrel and forelimb sensory cortex. NeuroImage 1:181-190.

Ogawa S, Lee TM, Kay AR, Tank DW (1990) Brain magnetic resonance imaging with contrast dependent on blood oxygenation. Proc Natl Acad Sci USA 87:9868-9872.

Ogawa S, Tank DW, Menon R, Ellermann JM, Kim S-G, Merkle H, Ugurbil K (1992) Intrinsic signal changes accompanying sensory stimulation: functional brain mapping with magnetic resonance imaging. Proc Natl Acad Sci USA 89:5951-5955.

Ogawa S, Menon RS, Tank DW, Kim SG, Merkle H, Ellermann JM, Ugurbil K (1993) Functional brain mapping by blood oxygenation level-dependent contrast magnetic resonance imaging. A comparison of signal characteristics with a biophysical model. Biophys J 64:803-812.

Pauling L (1977) Magnetic properties and structure of oxyhemoglobin. Proc Natl Acad Sci USA 74:2612-2613.

Paxinos G (1995) The rat nervous system. London: Academic.

Peterson BE, Goldreich D, Merzenich MM (1998) Optical imaging and electrophysiology of rat barrel cortex. I. Responses to small singlevibrissa deflections. Cereb Cortex 8:173-183.

Ratzlaff EH, Grinvald A (1991) A tandem-lens epifluorescence macroscope: hundred-fold brightness advantage for wide-field imaging. J Neurosci Methods 36:127-137.

Richter K, Hess A, Scheich H (1999) Functional mapping of transsynaptic effects of local manipulation of inhibition in gerbil auditory cortex. Brain Res 831:184-199.

Scheich H, Heil P, Langner G (1993) Functional organization of auditory cortex in the mongolian gerbil (Meriones unguiculatus). II. Tonotopic 2-deoxyglucose. Eur J Neurosci 5:898-914.

Scheich H, Baumgart F, Gaschler-Markefski B, Tegeler C, Tempelmann C, Heinze HJ, Schindler F, Stiller D (1998) Functional magnetic resonance imaging of human auditory cortex area involved in foregroundbackground decomposition. Eur J Neurosci 10:803-809.

Schwarzbauer C, Heinke W (1999) Investigating the dependence of BOLD contrast on oxidative metabolism. Magn Reson Med 41:537-543.

Thomas H, Tillein J, Heil P, Scheich H (1993) Functional organization of auditory cortex in the mongolian gerbil (Meriones unguiculatus ). I. Electrophysiological mapping of frequency representation and distinction of fields. Eur J Neurosci 5:882-897.

Thulborn KR, Waterton JC, Matthews PM, Radda GK (1982) Oxygenation dependence of the transverse relaxation time of water protons in whole blood at high field. Biochim Biophys Acta 714:265-270.

Turner R, Howseman A, Rees GE, Josephs O, Friston K (1998) Functional magnetic resonance imaging of the human brain: data acquisition and analysis. Exp Brain Res 123:5-12.

Ugurbil K, Hu X, Chen W, Zhu XH, Kim SG, Georgopoulos A (1999) Functional mapping in the human brain using high magnetic fields. Philos Trans R Soc Lond B Biol Sci 354:1195-1213.

Welker C (1976) Receptive fields of barrels in the somatosensory neocortex of the rat. J Comp Neurol 166:173-189.

Welker E, Rao SB, Dorfl J, Melzer P, van der Loos H (1992) Plasticity in the barrel cortex of the adult mouse: effects of chronic stimulation upon deoxyglucose uptake in the behaving animal. J Neurosci 12:153-170.

Wong-Riley MT, Welt C (1980) Histochemical changes in cytochrome oxidase of cortical barrels after vibrissal removal in neonatal and adult mice. Proc Natl Acad Sci USA 77:2333-2347.

Woolsey TA, Welker C, Schwartz RH (1975) Comparative anatomical studies of the SmL face cortex with special reference to the occurrence of "barrels" in layer IV. J Comp Neurol 164:79-94.

Yang X, Hyder F, Shulman RG (1996) Activation of single whisker barrel in rat brain localized by functional magnetic resonance imaging. Proc Natl Acad Sci USA 93:475-478.

Yang X, Hyder F, Shulman RG (1997) Functional MRI BOLD signal coincides with electrical activity in the rat whisker barrels [in process citation]. Magn Reson Med 38:874-877. 